\title{
Utility of endobronchial ultrasound-guided transbronchial needle aspiration in patients with tuberculous intrathoracic lymphadenopathy: a multicentre study
}

\author{
Neal Navani, ${ }^{1}$ Philip L Molyneaux, ${ }^{2}$ Ronan A Breen, ${ }^{3}$ David W Connell, ${ }^{2}$ \\ Annette Jepson, ${ }^{4}$ Matthew Nankivell, ${ }^{5}$ James M Brown, ${ }^{1}$ Stephen Morris-Jones, ${ }^{6}$ \\ Benjamin Ng, ${ }^{7}$ Melissa Wickremasinghe, ${ }^{2}$ Ajit Lalvani, ${ }^{2}$ Robert C Rintoul, ${ }^{7}$ \\ George Santis, ${ }^{3}$ Onn Min Kon, ${ }^{2}$ Sam M Janes ${ }^{1}$
}

\begin{abstract}
${ }^{1}$ Centre for Respiratory
Research, University College London, London, UK

${ }^{2}$ Respiratory Medicine, Imperial College Healthcare NHS Trust, London, UK

${ }^{3}$ Department of Thoracic Medicine, King's Health

Partners, London, UK

${ }^{4}$ Department of Microbiology, Imperial College Healthcare NHS Trust, London, UK

${ }^{5}$ MRC Clinical Trials Unit, London, UK

${ }^{6}$ Department of Microbiology, University College Hospital, London, UK

${ }^{7}$ Department of Thoracic Oncology, Papworth Hospital, Cambridge, UK
\end{abstract}

\section{Correspondence to}

Dr Sam Janes, Centre for Respiratory Research, University College London, 5 University Street, London WC1E 6JJ, UK; s.janes@ucl.ac.uk

NN and PM are joint first authors.

OMK and SMJ are joint senior authors.

Received 20 February 2011 Accepted 5 July 2011 Published Online First 3 August 2011

\begin{abstract}
Background Endobronchial ultrasound-guided transbronchial needle aspiration (EBUS-TBNA) has emerged as an important tool for the diagnosis and staging of lung cancer but its role in the diagnosis of tuberculous intrathoracic lymphadenopathy has not been established. The aim of this study was to describe the diagnostic utility of EBUS-TBNA in patients with intrathoracic lymphadenopathy due to tuberculosis (TB). Methods 156 consecutive patients with isolated intrathoracic TB lymphadenitis were studied across four centres over a 2-year period. Only patients with a confirmed diagnosis or unequivocal clinical and radiological response to antituberculous treatment during follow-up for a minimum of 6 months were included. All patients underwent routine clinical assessment and a CT scan prior to EBUS-TBNA. Demographic data, HIV status, pathological findings and microbiological results were recorded.
\end{abstract}

Results EBUS-TBNA was diagnostic of TB in 146 patients (94\%; 95\% Cl 88\% to 97\%). Pathological findings were consistent with TB in 134 patients (86\%). Microbiological investigations yielded a positive culture of TB in 74 patients (47\%) with a median time to positive culture of 16 days (range 3-84) and identified eight drug-resistant cases (5\%). Ten patients (6\%) did not have a specific diagnosis following EBUS; four underwent mediastinoscopy which confirmed the diagnosis of TB while six responded to empirical antituberculous therapy. There was one complication requiring an inpatient admission.

Conclusions EBUS-TBNA is a safe and effective firstline investigation in patients with tuberculous intrathoracic lymphadenopathy.

\section{INTRODUCTION}

The global threat of tuberculosis (TB) remains undiminished with the WHO estimating there were 9.4 million incident cases worldwide in 2009. ${ }^{1}$ The incidence in the UK has risen year on year over the last two decades, and this trend continued in 2009 with a $4.2 \%$ rise. Successful chemotherapy requires a combination of drugs for at least 6 months, but this may need to be substantially increased if resistance to the first-line agents is present. Indeed, the emergence of drug-resistant,

\section{Key messages}

What is the key question?

- What is the diagnostic utility of EBUS-TBNA in patients with tuberculous intra-thoracic lymphadenopathy?

\section{What is the bottom line?}

- EBUS-TBNA is an important and safe investigation for patients with tuberculous intra-thoracic lymphadenopathy. In 156 patients with intrathoracic lymph node TB, EBUS-TBNA was diagnostic in $94 \%$ of patients, provided a positive culture in $47 \%$ and identified 8 cases of drug resistant tuberculosis.

\section{Why read on?}

- Bronchoalveolar lavage and sputum culture have a low yield in patients with isolated tuberculous intra-thoracic lymphadenopathy. This multi-centre study is the first major description of the high diagnostic yield of EBUSTBNA in patients with tuberculous intra-thoracic lymphadenopathy.

multidrug-resistant and extremely drug-resistant TB over the last 20 years has emphasised the importance of establishing the correct diagnosis and drug susceptibilities of the mycobacterium before starting antituberculous therapy. ${ }^{2}$

While the number of cases of pulmonary TB has fallen in many developed countries over recent years, the notification of extrapulmonary disease has increased. In both the USA and UK, tuberculosis lymphadenitis (TBLA) is the commonest extrapulmonary manifestation among all ethnic groups. ${ }^{3}$ Mediastinal TBLA represented $9 \%$ of cases reported in the UK in $2009^{4}$ and presents significant diagnostic challenges. Clarifying the aetiology of isolated mediastinal lymphadenopathy is essential to exclude alternative diagnoses such as lymphoma, carcinoma and sarcoidosis. However, the lack of specific clinical and radiological features necessitates a pathological or microbiological diagnosis whenever possible. Mediastinal lymph node sampling is commonly performed by conventional 
transbronchial needle aspiration (TBNA), endoscopic ultrasound-guided fine needle aspiration or mediastinoscopy.

Endobronchial ultrasound-guided transbronchial needle aspiration (EBUS-TBNA) has now emerged as an important tool for the diagnosis of mediastinal and hilar lymphadenopathy. In addition to the nodal stations accessible by conventional TBNA, EBUS guidance also allows safe aspiration of hilar nodes and nodes $<10 \mathrm{~mm}$. In patients with lung cancer and sarcoidosis, EBUS-TBNA has been shown to increase the yield and sensitivity compared with standard bronchoscopic techniques including conventional TBNA. ${ }^{5-7}$ However, the role of EBUS-TBNA in the diagnosis of intrathoracic TBLA has not been established. In this multicentre study we describe the diagnostic utility of EBUS-TBNA for the diagnosis of intrathoracic TBLA.

\section{METHODS \\ Patients}

Consecutive patients with intrathoracic lymph node TB who were referred for EBUS-TBNA were included in the study. A final diagnosis of intrathoracic TBLA was confirmed by positive pathology, microbiology or an unequivocal clinical and radiological response to antituberculous treatment at least 6 months after presentation by the referring physician. Patients were excluded if sputum or bronchial washings were positive for acidfast bacilli on either smear or culture prior to EBUS-TBNA, or if the diagnosis was available from sampling of extrathoracic disease. Demographic data and HIV status were recorded. All patients were followed up for at least 6 months. The participating centres were University College Hospital (London), St Mary's Hospital (London), Guy's and St Thomas' Hospital (London) and Papworth Hospital (Cambridgeshire). Informed consent was obtained from each patient prior to undergoing EBUS-TBNA. The observational nature of the study meant that ethical approval was not required.

\section{Intervention}

Following contrast-enhanced thoracic CT scanning to assess the size and location of the lymphadenopathy, EBUS-TBNA was carried out in outpatients under local anaesthesia and moderate sedation using midazolam and fentanyl. The procedure was performed with an echo-bronchoscope (BF-UC160F-OL8 Olympus, Tokyo) which allows endoscopic views and simultaneous linear ultrasound of mediastinal and hilar structures. The location, number and size of the TBLA were recorded. Vascular structures were confirmed using the colour Doppler function. A dedicated aspiration needle (22 or 21 gauge) was then placed in the working channel and advanced into the lymph node under ultrasound guidance. Once the tip of the needle was visualised in the lymph node, the stylet was withdrawn and suction applied to the needle and the needle was then moved to and fro within the lymph node. Two centres employed on-site evaluation of the cell content of samples which determined the number of passes. Where on-site evaluation was not available, at least three passes per lymph node were obtained. Smears were prepared directly onto slides for cytological analysis. Samples were also expelled directly into formalin for cell block analysis. The needle contents from at least one dedicated pass were submitted in saline for microbiological analysis. The microbiological specimens were analysed by fluorescence microscopy using direct auramine stains and Middlebrook 7H9 medium (an element of the BACTEC MGIT 960 System) was used to culture Mycobacterium spp.

\section{Assessment of samples}

Pathological findings were classified into five grades as documented previously ${ }^{8}$ : grade I: epithelioid granulomatous reaction with caseation; grade II: epithelioid granulomatous reaction without caseation; grade III: non-granulomatous reaction with necrosis; grade IV: non-specific; and grade V: inadequate sample. Grades I-III were considered to be consistent with a diagnosis of intrathoracic TBLA in the context of clinical features, supportive tuberculin skin test or interferon $\gamma$ release assay and a clinical response to treatment. Microbiological investigations were considered positive for TB if the smear was positive for acid-fast bacilli or culture-isolated Mycobacterium tuberculosis.

\section{Statistical analysis}

The standard definition for diagnostic sensitivity was employed and is the percentage of patients in the study diagnosed with TB by EBUS-TBNA. Since the prevalence of TB in the cohort was $100 \%$, predictive values were not calculated. Predictors of a positive culture for TB were modelled using logistic regression. Continuous variables were not categorised in the regression analyses. Significant variables in univariate analysis (at the $10 \%$ level) or those deemed clinically important were included in the multivariate model. Interactions were assessed for all covariates considered for inclusion in the multivariate analysis by including interaction variables in the multivariate model to see if the model could be improved. Analyses were conducted using Stata Version 10 (Stata Corporation, USA). This study and its report conforms to the standards for the reporting of diagnostic accuracy studies (STARD) statement. ${ }^{9}$

\section{RESULTS}

Between 1 January 2008 and 1 February 2010, 156 consecutive patients who subsequently received a final diagnosis of TBLA underwent EBUS-TBNA at four centres. The median age at the time of the procedure was 39 years (range 18-86). There were 80 men $(51 \%)$. The most common clinical symptom was cough in 94 patients (60\%). Other presenting symptoms included weight loss, cough, haemoptysis and night sweats and are summarised in table 1.

At EBUS, mediastinal and hilar lymph nodes of median size $22 \mathrm{~mm}$ (range 5-60 $\mathrm{mm}$ ) were detected. The subcarinal lymph node station (station 7) was the most common location for EBUS-guided sampling (44\% of nodes sampled), followed by the

Table 1 Baseline characteristics

\begin{tabular}{ll}
\hline Total number of patients & 156 \\
Male & $80(51 \%)$ \\
Median age (range) & $39(18-86)$ \\
Ethnicity & \\
$\quad$ African & $47(30 \%)$ \\
Caucasian & $25(16 \%)$ \\
Caribbean & $6(4 \%)$ \\
South Asian & $56(36 \%)$ \\
East Asian & $12(8 \%)$ \\
Other & $10(6 \%)$ \\
Presenting symptoms & \\
Fever/night sweats & $76(49 \%)$ \\
Weight loss & $72(46 \%)$ \\
Cough & $94(60 \%)$ \\
Haemoptysis & $12(77 \%)$ \\
$\quad$ No symptoms & $34(22 \%)$ \\
Abnormal lung parenchyma on CT & $54(35 \%)$ \\
HIV-positive & $17(11 \%)$ \\
\hline
\end{tabular}


Table 2 Results for endobronchial ultrasound-guided transbronchial needle aspiration (EBUS-TBNA) of 220 lymph nodes in 156 patients with intrathoracic tuberculous lymphadenopathy according to lymph node location

\begin{tabular}{llll}
\hline $\begin{array}{l}\text { Lymph } \\
\text { node } \\
\text { station* }\end{array}$ & $\begin{array}{l}\text { Number of } \\
\text { nodes sampled } \\
\text { at EBUS-TBNA }\end{array}$ & $\begin{array}{l}\text { Number of nodes from } \\
\text { which pathological } \\
\text { grades I-III were } \\
\text { obtained }\end{array}$ & $\begin{array}{l}\text { Number of nodes } \\
\text { from which positive } \\
\text { culture for tuberculosis } \\
\text { was obtained }\end{array}$ \\
\hline 2R & 3 & 3 & 0 \\
2L & 1 & 1 & 0 \\
4R & 63 & 54 & 27 \\
4L & 13 & 11 & 7 \\
7 & 96 & 87 & 53 \\
10R & 28 & 26 & 10 \\
10L & 13 & 10 & 5 \\
11R & 1 & 1 & 1 \\
11L & 2 & 2 & 1 \\
\hline
\end{tabular}

${ }^{*}$ According to Mountain-Dresler lymph node map.

right paratracheal lymph node station (4R) (29\% of nodes sampled; table 2). Sixty-one patients (39\%) had two or more nodal stations sampled.

EBUS-TBNA was diagnostic of TB in 146 patients (94\%, 95\% CI $88 \%$ to 97\%). Pathological findings were consistent with TB in 134 patients (86\%); 68 (44\%) had granulomas with necrosis, $58(37 \%)$ had granulomas without necrosis and eight $(5 \%)$ had necrosis alone. Nineteen patients (12\%) had lymphocytes alone from EBUS-TBNA and in three patients $(2 \%)$ the sample was inadequate for pathological assessment.

Microbiological investigations of EBUS-TBNA yielded a diagnosis of TB in 82 patients (53\%). In 27 patients (17\%) the smear of the EBUS-guided aspirate was positive for acid-fast bacilli. Seventy-four patients (47\%) had a positive culture for $M$ tuberculosis with a median time to positive culture of 16 days (range 3-84). In eight patients (5\%) the EBUS aspirate stained positive for acid-fast bacilli but the culture was negative. Eight patients (5\%) were proven to have isoniazid-resistant TB. In 15 patients $(10 \%)$ the pathology was negative but a firm diagnosis of TB was obtained on auramine/Ziehl-Neelsen stain or culture. In the logistic regression model for predicting positive TB culture, age, ethnicity, lymph node size, lymph node location, HIV status, lung parenchymal appearances, number of lymph nodes sampled and the number of needle passes per node were analysed (table 3). Pathology showing necrosis was considered a clinically important variable to be included in the multivariate model. The presence of necrosis on EBUS-TBNA pathology and sampling more than one lymph node were both significant at the $10 \%$ level in the multivariate regression model and may be associated with a positive culture (table 3). A significant interaction at the $10 \%$ level between lymph node size and necrosis on pathology was observed with a positive culture less likely to occur in larger nodes with necrosis ( $\mathrm{p}=0.074$; OR $0.292,95 \% \mathrm{CI}$ 0.075 to 1.128$)$. No other significant interactions were identified. Ten patients $(6 \%)$ did not have a specific diagnosis following EBUS-TBNA. Of these, four underwent mediastinoscopy which confirmed the diagnosis of TB in all cases, while six patients received empirical antituberculous therapy (figure 1).

One patient undergoing EBUS-TBNA experienced a serious complication necessitating inpatient admission. The patient was a 32-year-old man of south Asian origin in whom a $35 \mathrm{~mm}$ right paratracheal lymph node was aspirated under EBUS guidance. Four uncomplicated passes into the lymph node were made and the procedure yielded necrotising granulomas which were also positive for acid-fast bacilli on Ziehl-Neelsen staining. Antituberculous therapy was initiated. However, 2 days after the procedure the patient presented with sepsis and blood cultures were positive for a $\beta$-haemolytic group $G$ streptococcus. The patient improved with appropriate antibiotics and was discharged on the eighth day after the procedure without further complications. The episode was ascribed to insertion of the bronchoscope itself rather than performance of TBNA, as previously described. ${ }^{10}$

\section{DISCUSSION}

This is the first study to assess the utility of EBUS-TBNA in the diagnosis of intrathoracic TBLA and demonstrates a sensitivity of $94 \%$ for the technique in 156 patients, with one complication observed. In 74 patients (47\%) a positive culture of $M$ tuberculosis was obtained.

In patients with isolated mediastinal lymphadenopathy due to $\mathrm{TB}$, traditional techniques of bronchoscopy and sputum culture have a low yield for positive culture. ${ }^{11}$ Mediastinoscopy may be employed but requires general anaesthesia, carries a morbidity of $1-2 \%$ and also has the disadvantage that posterior subcarinal and hilar nodes are inaccessible. Recently, endoscopic ultrasound-guided fine needle aspiration (EUS-FNA) of tuberculous mediastinal lymphadenopathy has been described. ${ }^{12} 13$ In these studies the diagnostic yield was $90-93 \%$. However, EUS-FNA does not allow access to the right paratracheal and hilar lymph node stations which are commonly involved in $\mathrm{TB}^{11}$ and accounted for $47 \%$ of the nodal stations sampled in this study.

EBUS-TBNA now provides an important alternative in patients with intrathoracic TBLA. The procedure is well tolerated in the outpatient setting, provides access to the mediastinal

Table 3 Univariate and multivariate analyses of factors to predict positive culture of Mycobacterium tuberculosis in patients undergoing endobronchial ultrasound-guided transbronchial needle aspiration

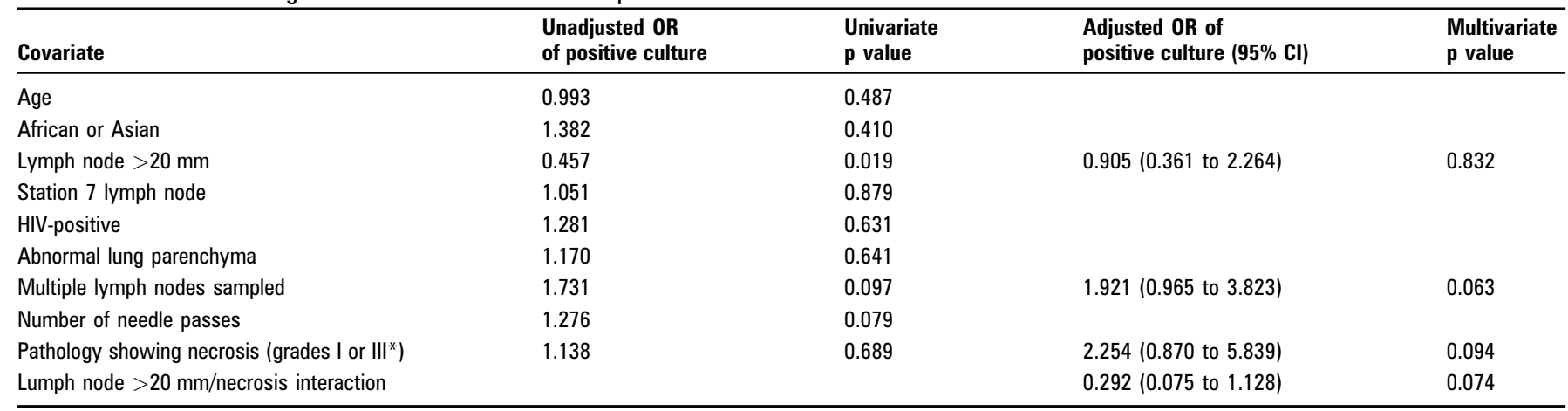

*Grade I, epithelioid granulomatous reaction with caseation; grade III, granulomatous reaction with necrosis. 
Figure 1 Flowchart of patients with intrathoracic lymph node tuberculosis (TBLA) undergoing endobronchial ultrasound-guided transbronchial needle aspiration (EBUS-TBNA). M.tb, Mycobacterium tuberculosis.

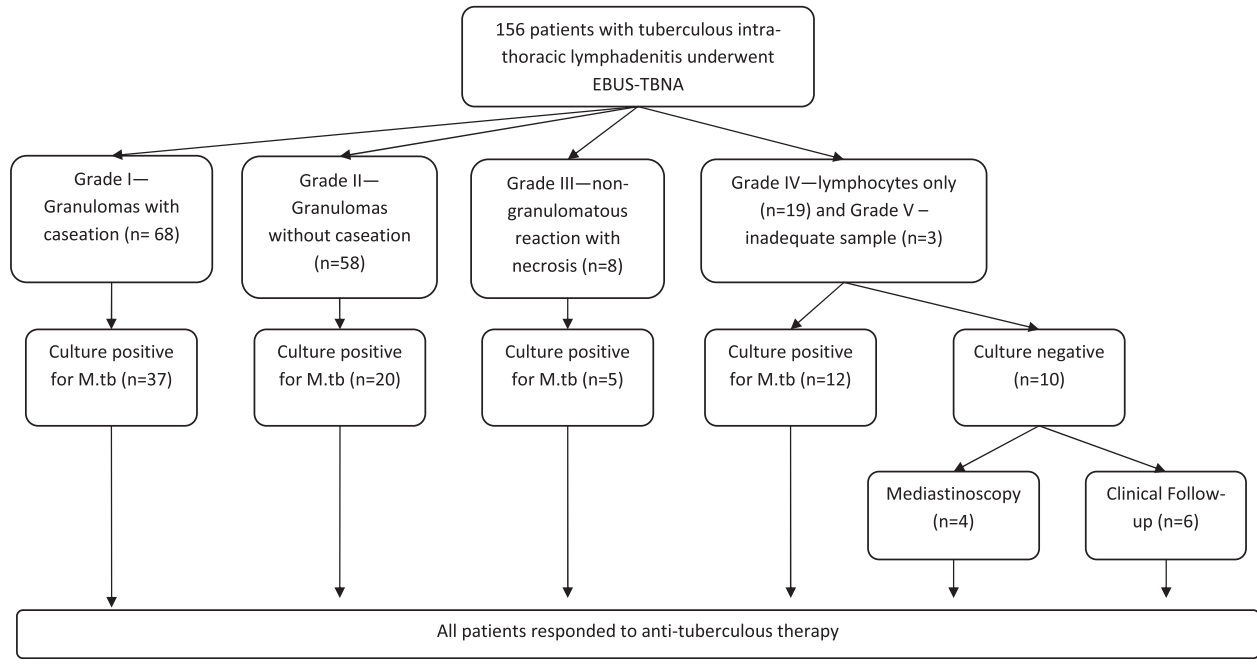

and hilar lymph node locations commonly involved in TB and also allows bronchial washings to be performed at the same procedure. Successful isolation of an organism allows susceptibility testing, which is an increasingly necessary clinical need in the UK, as elsewhere, given the prevalence of isoniazid-resistant and multidrug-resistant disease. ${ }^{4}$ Prior to the advent of EBUSTBNA, many of these patients would have received empirical antituberculous therapy. However, demonstration of a resistant organism ( $>10 \%$ of the culture-positive patients in this cohort) significantly alters the antituberculous regimen and duration of treatment, ${ }^{14}$ and suboptimal treatment may induce selection of further drug-resistant strains. In addition, it is well recognised that intrathoracic TBLA may not alter radiologically on successful treatment and, in fact, a significant proportion may paradoxically increase during treatment. In this setting, a firm microbiological diagnosis avoids the risk of inappropriate antimicrobial escalation but, instead, allows consideration of the use of anti-inflammatory treatments.

The culture rate of $47 \%$ observed in this study is similar to culture rates from lymph node sampling observed with other modalities. In a study of 29 patients with intrathoracic TBLA who underwent mediastinoscopy, 14 (48\%) had a positive culture for $M$ tuberculosis. ${ }^{15}$ Bilaceroglu et al ${ }^{16}$ report a culture rate of $26 \%$ (17/63) performing TBNA without EBUS while, in a study of EUS, the culture rate was $21 \%{ }^{13}$ Gupta and colleagues $^{17}$ obtained a positive culture for TB in $49 \%$ of lymph node fine needle aspirates. Gulati et al ${ }^{18}$ demonstrated that TB was cultured in seven out of 26 patients undergoing US-guided percutaneous mediastinal lymph node biopsies. These low culture rates are likely to represent the heterogeneity in bacillary load of intrathoracic tuberculous lymph nodes and the yield obtained with EBUS is comparable with these other modalities. The mean time to culture was 16 days (range 3-84). One centre extended liquid mycobacterial culture beyond 6 weeks' incubation and identified three additional isolates. Further investigation into the value of extended culture and of the potential application of rapid molecular techniques, such as the GeneXpert MTB platform, ${ }^{19}$ is warranted to try to further increase sensitivity.

The logistic regression model showed that those EBUS procedures which obtained necrotic granulomas or necrosis alone were more likely to have a positive culture for TB. It may be postulated that the bacillary load in these lymph nodes is higher in order to cause necrosis and therefore the organism in these patients is more likely to be cultured. As has been previously demonstrated in patients with sarcoidosis undergoing conventional $\mathrm{TBNA}^{20}$ sampling more than one lymph node station increases the diagnostic yield. Although many patients were observed to have matted and hypoechoic lymph nodes on endobronchial ultrasound views, the significance of these findings was not assessed.

Seventeen patients included in the study were infected with HIV and culture was positive in six of these patients. A previous report has shown that EBUS-TBNA may diagnose non-tuberculous mycobacterial disease in a patient with AIDS. ${ }^{21}$ Further data are required on the utility of EBUS-TBNA in HIV-infected individuals.

Data from a systematic review of patients with non-small cell lung cancer undergoing EBUS-TBNA indicate that EBUS-TBNA is a safe procedure with minimal complications. ${ }^{22}$ In this report we describe one complication of symptomatic bacteraemia following EBUS-TBNA of necrotic mediastinal lymphadenopathy. Von Bartheld and colleagues ${ }^{23}$ have described the formation of a mediastinal-oesophageal fistula in a patient following EUS-FNA with a 22 gauge needle of a heterogeneous subcarinal gland. There has also been a report of mediastinitis following EUS-guided aspiration of necrotic subcarinal lymph node in a patient with non-small cell lung cancer. ${ }^{24}$ It may be postulated that the risk of infectious complications may be increased in patients undergoing aspiration of large necrotic lymph nodes and further safety data are required in this patient group.

Limitations of the current study are recognised. TB has a high endemic rate in our predominantly London-based population with an incidence of 44.3 per 100000 per year. ${ }^{4}$ In addition, the centres included in the study have considerable experience with EBUS-TBNA ${ }^{25}$ so the results may not be applicable to other areas. The retrospective nature of this study prevented the inclusion of patients with isolated mediastinal lymphadenopathy and therefore it is not possible to determine the disease prevalence of TB in our population undergoing EBUS-TBNA. In this study, the finding of granulomas without caseation along with supporting clinical evidence and response to therapy was Although a positive tuberculin skin test or interferon $\gamma$ release assay adds weight to the diagnosis of $\mathrm{TB}$, it is possible that these investigations may still be positive in patients with sarcoidosis from populations such as ours with a high incidence of TB. Such patients may also clinically improve spontaneously and be classed inappropriately as responders to antituberculous treatment. However, even allowing for this, it should be noted that in considered to be consistent with a final diagnosis of $\mathrm{TB}$. 
the group with non-caseating granulomas, 20 of the 58 cases with this cytology were culture-positive (in addition to clinical criteria of improvement on treatment), indicating that this pathological finding is in itself still compatible with active TB. Until it is clear if the use of other diagnostic tools (such as molecular tests) allows further refinement of the diagnosis, this common clinical conundrum of commencing treatment for TB in the setting of a culture-negative mediastinal node with supportive clinical and epidemiological features remains.

In conclusion, EBUS-TBNA is a safe and effective first-line investigation in patients with intrathoracic TBLA.

Acknowledgements We would like to thank the patients in this study and acknowledge the EBUS for Tuberculosis Interest Group: St Mary's, Imperial College Healthcare NHS Trust: Sarah Murthy (HIV), Graham Cooke (HIV), Corinna Wright (Cytology), Peter George (Respiratory) and Aran Singanayagam (Respiratory). University College Hospital NHS Trust: Helen L Booth (Respiratory), Robert F Miller (HIV), Gabrijela Kocjan (Pathology), Arrigo Capitanio (Pathology), Mary Falzon (Pathology) and Ali Zumla (Infectious Diseases).

Funding NN is an MRC Clinical Research Training Fellow. SMJ is a Wellcome Trust Senior Fellow in Clinical Science. This study was partly undertaken at University College London Hospital/University College London who received a proportion of funding from the Department of Health's NIHR Biomedical Research Centre's funding scheme (NN, SMJ, SM-J). This study was also partly undertaken at St Mary's Hospital, Imperial College Healthcare NHS Trust which is supported by the NIHR Biomedical Research Centre funding scheme and the Centre for Respiratory Infection at Imperial College London, supported by the Wellcome Trust and partly undertaken at Papworth Hospital, Cambridge who are funded by the Department of Health's NIHR Biomedical Research Centre's funding scheme (RCR) and the Cambridge Experimental Cancer Medicines Centre.

\section{Competing interests None.}

Contributors NN, PM: conception of project, performance of procedures, compilation and analysis of data, production of manuscript. RAB, DC, MW, RCR, GS: performance of procedures, review of manuscript. AJ, SM-J: microbiology assessment, review of manuscript. MN: analysis of data, review of manuscript. JMB: performance of procedures, analysis of data, review of manuscript. BN: compilation of data, review of manuscript. AL: review of manuscript. OMK, SMJ: conception of project, performance of procedures, review of manuscript.

Provenance and peer review Not commissioned; externally peer reviewed.

\section{REFERENCES}

1. World Health Organization. Global tuberculosis control 2010. 2010. http:// whqlibdoc.who.int/publications/2010/9789241564069 eng.pdf.

2. World Health Organization. Multidrug and extensively drug-resistant TB (M/XDRTB): 2010 Global report on surveillance and response. 2010. http://whqlibdoc.who. int/publications/2010/9789241599191 eng.pdf.

3. Fiske CT, Griffin MR, Erin H, et al. Black race, sex, and extrapulmonary tuberculosis risk: an observational study. BMC Infect Dis 2010;10:16.
4. Health Protection Agency. Tuberculosis in the UK 2010. 2010. http://www.hpa. org.uk/web/HPAwebFile/HPAweb_C/1287143594275.

5. Wallace MB, Pascual JM, Raimondo M, et al. Minimally invasive endoscopic staging of suspected lung cancer. JAMA 2008;299:540-6.

6. Tremblay A, Stather DR, Maceachern P, et al. A randomized controlled trial of standard vs endobronchial ultrasonography-guided transbronchial needle aspiration in patients with suspected sarcoidosis. Chest 2009;136:340-6.

7. Navani N, Booth HL, Kocjan G, et al. Combination of endobronchial ultrasound guided transbronchial needle aspiration with standard bronchoscopic techniques for the diagnosis of stage I and stage II pulmonary sarcoidosis. Respirology 2011:16:467-72.

8. Bezabih M, Mariam DW, Selassie SG. Fine needle aspiration cytology of suspected tuberculous lymphadenitis. Cytopathology 2002;13:284-90.

9. Bossuyt PM, Reitsma JB, Bruns DE, et al. Towards complete and accurate reporting of studies of diagnostic accuracy: the STARD initiative. BMJ 2003;326:41-4.

10. Steinfort DP, Johnson DF, Irving LB. Incidence of bacteraemia following endobronchial ultrasound-guided transbronchial needle aspiration. Eur Respir $J$ 2010;36:28-32.

11. Codecasa LR, Besozzi G, De CL, et al. Epidemiological and clinical patterns of intrathoracic lymph node tuberculosis in 60 human immunodeficiency virus-negative adult patients. Monaldi Arch Chest Dis 1998;53:277-80.

12. Puri R, Vilmann P, Sud R, et al. Endoscopic ultrasound-guided fine-needle aspiration cytology in the evaluation of suspected tuberculosis in patients with isolated mediastinal lymphadenopathy. Endoscopy 2010;42:462-7.

13. Song HJ, Park YS, Seo DW, et al. Diagnosis of mediastinal tuberculosis by using EUS-guided needle sampling in a geographic region with an intermediate tuberculosis burden. Gastrointest Endosc 2010;71:1307-13.

14. National Institute for Health and Clinical Excellence (NICE). NICE guidelines on tuberculosis. 2006. http://www.nice.org.uk/CG33.

15. Farrow PR, Jones DA, Stanley PJ, et al. Thoracic lymphadenopathy in Asians resident in the United Kingdom: role of mediastinoscopy in initial diagnosis. Thorax 1985; 40:121-4.

16. Bilaceroglu S, Gunel O, Eris N, et al. Transbronchial needle aspiration in diagnosing intrathoracic tuberculous lymphadenitis. Chest 2004:126:259-67.

17. Gupta SK, Chugh TD, Sheikh ZA, et al. Cytodiagnosis of tuberculous lymphadenitis. A correlative study with microbiologic examination. Acta Cytol 1993;37:329-32.

18. Gulati M, Venkataramu NK, Gupta S, et al. Ultrasound guided fine needle aspiration biopsy in mediastinal tuberculosis. Int J Tuberc Lung Dis 2000;4:1164-8.

19. Boehme CC, Nabeta P. Hillemann D, et al. Rapid molecular detection of tuberculosis and rifampin resistance. N Engl J Med 2010;363:1005-15.

20. Trisolini R, Tinelli C, Cancellieri A, et al. Transbronchial needle aspiration in sarcoidosis: yield and predictors of a positive aspirate. J Thorac Cardiovasc Surg 2008; 135:837-42.

21. Steinfort DP, Johnson DF, Connell TG, et al. Endobronchial ultrasound-guided biopsy in the evaluation of intrathoracic lymphadenopathy in suspected tuberculosis: a minimally invasive technique with a high diagnostic yield. J Infect 2009:58:309-11.

22. Gu P, Zhao YZ, Jiang $L Y$, et al. Endobronchial ultrasound-guided transbronchial needle aspiration for staging of lung cancer: a systematic review and meta-analysis Eur J Cancer 2009:45:1389-96.

23. von Bartheld MB, van Kralingen KW, Veenendaal RA, et al. Mediastinal-esophageal fistulae after EUS-FNA of tuberculosis of the mediastinum. Gastrointest EndosC 2010; 71:210-12

24. Aerts JG, Kloover J, Los J, et al. EUS-FNA of enlarged necrotic lymph nodes may cause infectious mediastinitis. J Thorac Oncol 2008;3:1191-3.

25. Navani N, Nankivell $M$, Nadarajan $\mathrm{P}$, et al. The learning curve for EBUS-TBNA. Thorax 2011;66:352-3. 